\title{
A PC-controlled microwave tomographic scanner for breast imaging
}

\author{
Shantanu Padhi, ${ }^{1, \text { a) }}$ John Howard,${ }^{1}$ A. Fhager, ${ }^{2}$ and Sebastian Bengtsson ${ }^{2}$ \\ ${ }^{1}$ PRL, Research School of Physics and Engineering, Australian National University, Canberra, \\ ACT 0200, Australia \\ ${ }^{2}$ Biomedical Engineering Division, Department of Signals and Systems, Chalmers University of Technology, \\ Göteborg, SE-41296 Sweden
}

(Received 6 July 2010; accepted 9 November 2010; published online 21 January 2011)

\begin{abstract}
This article presents the design and development of a personal computer based controller for a microwave tomographic system for breast cancer detection. The system uses motorized, dual-polarized antennas and a custom-made GUI interface to control stepper motors, a wideband vector network analyzer (VNA) and to coordinate data acquisition and archival in a local MDSPlus database. Both copolar and cross-polar scattered field components can be measured directly. Experimental results are presented to validate the various functionalities of the scanner. () 2011 American Institute of Physics. [doi:10.1063/1.3523048]
\end{abstract}

\section{INTRODUCTION}

Microwave imaging of the complex permittivity profile of an unknown object placed inside a dielectric interface is an important technology that has been used successfully in subsurface sensing such as Ground Penetrating Radar (GPR) applications, land mine detection ${ }^{1}$ and nondestructive testing such as detection of defects and cracks in construction materials. ${ }^{2-5}$ This approach has received considerable attention due to its potential application in detection of breast cancer.

Research on the microwave dielectric properties of various human tissues has demonstrated that the electrical properties of normal and malignant human breast tissues differ by 5-10-fold over a frequency range of hundreds of megahertz to a few gigahertz. ${ }^{6,7}$ Microwave imaging techniques use this contrast between the malignant and soft tissues to image the breast tissue compared to more established techniques, such as x-ray tomography. Presently, two different approaches are used to detect the dielectric contrast between the object and the background medium (i) radar-like backscattering methods and (ii) tomographic methods. In radar-like methods, an ultrawideband signal illuminates the object from several directions, and the amplitude and/or the arrival time of the reflected signals are used to identify the location of the strong scatterer. ${ }^{8}$ This method has an advantage over the tomographic approach in managing the computational resources. However, this method lacks the accuracy in estimating the location of scatterer from time-of-flight measurement data which in principle requires a priori information of the dielectric media inside the region of interest. In the tomographic approach the measured scattering data are used to recover quantitative images of the dielectric profile using either linear or nonlinear algorithm. ${ }^{9-13}$

A number of microwave imaging measurement systems have been reported in the literature for data acquisition

\footnotetext{
a) Present address: Curtin Institute of Radio Astronomy (CIRA), ICRAR, Curtin University, Perth, WA-6102, Australia. Electronic mail: shantanu_padhi@ieee.org.
}

systems ${ }^{14,15}$ considering copolar scattered field components only. It has been well documented in electromagnetic theories that a thin dielectric cylinder of arbitrary cross-section and infinite length exhibits a large scattering cross-section in regards to the incident polarization along its length. As the tumor tissues are modelled as dielectric cylinders with finite length and placed in arbitrary directions, it is reasonable to expect strong copolar and cross-polar scattered electric fields with regard to input polarizations. It has been reported elsewhere ${ }^{16,17}$ that strong cross-polar scattered field components are seen in certain receiver directions. In our earlier work, ${ }^{18}$ we used an experimental system consisting of 20 monopoles of finite length, arranged in a circular grid of $200 \mathrm{~mm}$ diameter on a finite ground plane of $250 \mathrm{~mm}$ $\times 250 \mathrm{~mm}$. The copolar scattered data were collected in sequence, where each monopole worked as a transmitter and the others as receivers. In this article, we present a new experimental set-up for a microwave tomography measurement system. The system uses two dual-polarized antennas where one is a fixed transmitter, and the other is a receiver that can be placed at any angle to improve the angular sampling and spatial resolution. A dual polarized antenna is used to measure both copolar and cross-polar field components and the antenna can move vertically up and down for complete 3D measurements. This article is organized as follows: Section II describes the hardware system and the LABVIEW based control system and its integration with the MDSPlus database. ${ }^{19}$ First experimental results that compare the measured and modelled impedance and radiation patterns for dual polarized patch antennas are presented in Sec. III. And the conclusions are given in Sec. IV.

\section{SYSTEM AND INTERFACE DEVELOPMENT}

The microwave imaging system consists of following parts (i) a microwave scanner unit, (ii) two stepper motors, (iii) vector network analyzer (VNA), and (iv) a personal computer (PC) with LABVIEW controller. A 3D view of the scanning system is shown in Fig. 1. The microwave Scanner unit 


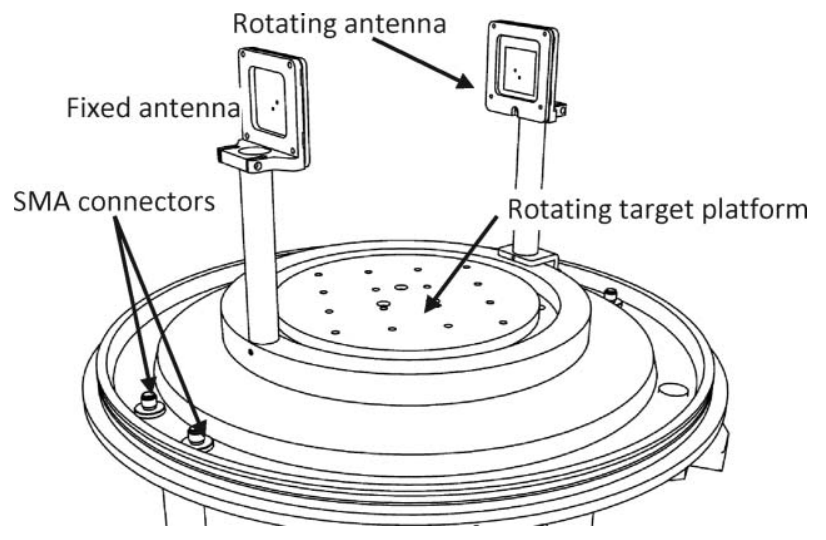

FIG. 1. A 3D-view of the microwave scanner unit.

consists of two movable outer and inner plates mounted on a base plate. The movable outer and inner plates are connected to two stepper motors (Sanyo Denki StepSyn motors) and are driven by two stepper motor drivers. The motors are placed below the base plate and the motor shafts feed through the base plate. The shafts and bearing were completely sealed by using water tight lip seal.

The motor controller unit and the VNA are connected through USB and GPIB (general purpose interface bus) cables, respectively. The receiver antenna is mounted on the movable outer plate and the transmitter antenna is mounted on fixed base plate. The height of both antennas are manually adjusted by using antenna holders of different length. At this stage the vertical movement of the antenna is carried out manually, however, this feature will be automated using another motor controller in our next generation experimental setup. A cylindrical Perspex (clear) container wall with a radius of $150 \mathrm{~mm}$ and a height of $250 \mathrm{~mm}$ is used to allow the antennas and the test object to be immersed in water or coupling fluid. To prevent leakage of coupling fluid, o-rings are extensively used around the base of the container wall, and hermetically sealed connectors are used to connect the RF cables. An outlet on the base plate allows water to be removed after performing the experiment.

A LABVIEW-based GUI (graphical user interface) has been developed to control the network analyzer and the stepper motors during the experiment, where the user runs and customizes different experiments. All experiment data and settings are saved into a single, self-descriptive hierarchical structure in an MDSplus database. Simple user friendly routines developed in intermediate dielectric layer and MATLAB access the data from the MDSPlus database server for postprocessing and further analysis.

\section{EXPERIMENTAL VALIDATION}

In order to validate the experimental performance of our tomographic system, we used two different data sets (impedance and radiation pattern of dual polarized circular patch antenna) for comparison. The measured S-parameter data and the radiation pattern (including gain) data of the dual polarized antenna are compared with the data from our 3D FDTD solver and the commercial software (CST-MWS ${ }^{\circledR}$ )
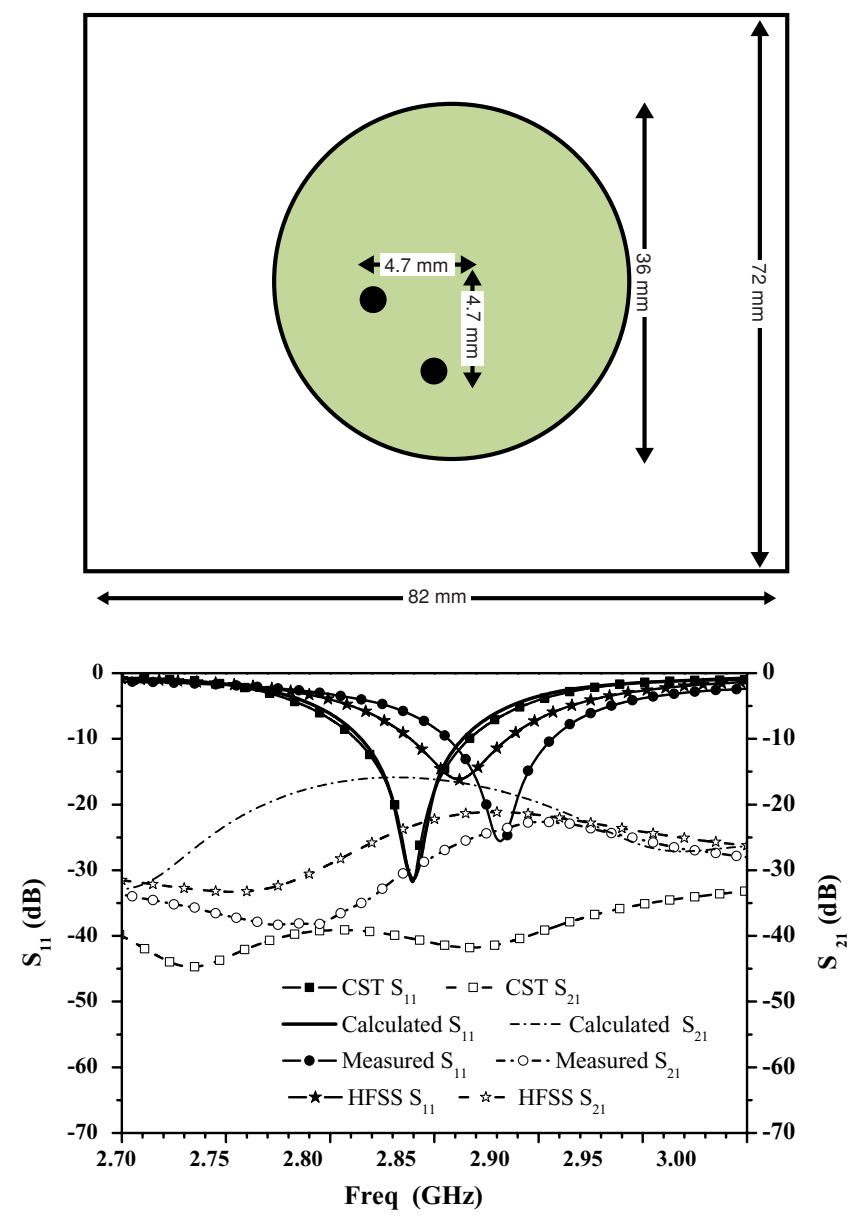

FIG. 2. (Color online) (a) The geometry of the dual polarized CMPA. (b) The calculated and measured S-parameters of the antenna.

CST Microwave Studio ${ }^{20}$ and Ansoft HFSS (high frequency structure simulation) ${ }^{21}$ respectively.

\section{A. Impedance behavior}

The geometry of a dual polarized circular microstrip patch antenna (CMPA) is shown in Fig. 2(a). The circular patch was constructed on $1.52 \mathrm{~mm}$ thick Arlon substrate (AR-270A) with material permittivity $\epsilon_{r}$ of 2.7 and loss tangent $(\tan \delta$ ) of 0.0018 (at $10 \mathrm{GHz}$ ). The other dimension details are given in Fig. 2. The antenna was fabricated using the combination of milling machine (Protomat S series) and chemical etching facilities available at ANU (The Australian National University). Two orthogonal polarizations were excited by using $1.1 \mathrm{~mm}$ diameter coaxial probes. The feed point was chosen at $4.7 \mathrm{~mm}$ offset from center for optimum match to the $50-\Omega$ input. For the finite-difference time domain (FDTD) calculation we used a uniform grid size of 0.5 $\mathrm{mm}$ and the computational domain was truncated by seven layers of CPML (convolutional PML). In 3D FDTD, the feed was modeled as a standard resistive voltage source (RVS) with internal resistance of $50-\Omega$. A Gaussian sinusoidal envelope (doublet) waveform with center frequency at $3 \mathrm{GHz}$ and full-width half-maximum (FWHM) of $3.0 \mathrm{GHz}$ was used as the excitation. A 2-port calibration was performed and the 
reference plane was deembedded to the end of the cables that connect the antennas. The calculated and measured Sparameters $\left(\mathrm{S}_{11}\right.$ and $\left.\mathrm{S}_{21}\right)$ of the dual polarized CMPA antenna is shown in Fig. 2(b) along with the calculated data using HFSS and CST.

As can be seen, there is a close agreement between the simulation and measurement. The measured resonant frequencies of the dual polarized antenna are $2.884 \mathrm{GHz}\left(\mathrm{S}_{11}\right)$ and $2.895 \mathrm{GHz}\left(\mathrm{S}_{22}\right)$ compared to the calculated (FDTD solver) resonant frequencies of $2.84 \mathrm{GHz}\left(\mathrm{S}_{11}\right)$ and $2.832 \mathrm{GHz}$ $\left(S_{22}\right)$, respectively. The return loss $(R L)$ results $\left(S_{11}\right)$ from FDTD solver compares very well with the data simulated with CST microwave studio. The FDTD calculated and measured impedance bandwidth (10 dB RL) is $1.76 \%$ and $1.74 \%$, respectively. The measured isolation between the two polarizations $\left(S_{21}\right)$ is better than $-23 \mathrm{~dB}$ compared to the calculated isolation (from FDTD solver) of $-16 \mathrm{~dB}$. The isolation between the two polarizations is better than $-33 \mathrm{~dB}$ in CST microwave studio. The calculated return loss and transmission loss data (isolations) from HFSS compare very well with the measured data. The HFSS predicted isolations are better than $-25 \mathrm{~dB}$ compared to measured value of $-23 \mathrm{~dB}$ over the fre-
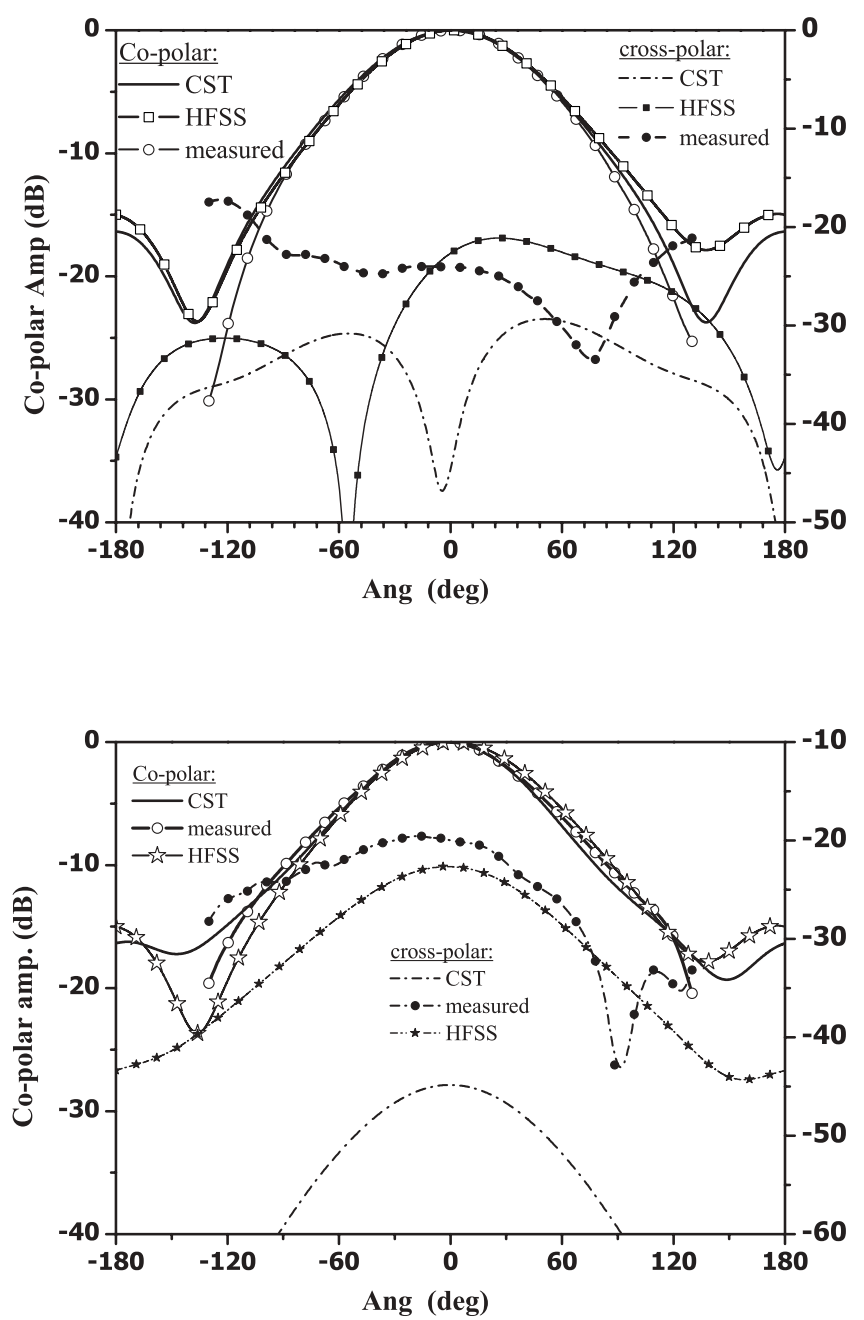

FIG. 3. (a) Co- and cross-polar radiations pattern of the CMPA in $\phi=0^{\circ}$ plane. (b) Co- and cross-polar radiation patterns of the CMPA in $\phi=90^{\circ}$ plane. quency band. The shift in resonant frequency (in $\mathrm{S}_{11}$ ) data) may be due to the combination of staircase features for circular shape used in FDTD grid and the finite inductance of the coaxial probes.

\section{B. Radiation pattern}

Radiation pattern measurements were performed with the microwave scanner from $-130^{\circ}$ to $+130^{\circ}$ in azimuth plane and the data were compared with the simulated data from CST and HFSS at resonant frequencies only. The calculated and measured radiation patterns for $\phi=0^{\circ}$ plane and $\phi=90^{\circ}$ plane are shown in Figs. 3(a) and 3(b) for single polarization only. As can be seen, the measured radiation patterns (both copolar and cross-polar) agree closely with HFSS simulated data. The measured cross-polar levels are very much consistent with the data shown in Fig. 2(b). Similar consistencies are found in HFSS data. For quantitative measurements, we compared the 3-dB beam-widths (and directivity) for both patterns. The calculated and measured beam-widths for $\phi=0^{\circ}$ plane and $\phi=90^{\circ}$ plane are $\left(84.4^{\circ}, 79.4^{\circ}\right)$ and $\left(84.44^{\circ}, 82.4^{\circ}\right)$, respectively. The calculated and measured directivity are 7.8 and $7.7 \mathrm{dBi}$, respectively.

\section{Imaging using cross-polar data}

In this experiment we would like to measure quantitatively the cross-polar power level of different target placed at different orientations for imaging. Two different targets, metallic and dielectric (perspex sheet with permittivity 1.1) targets were selected and placed at two different orientations. The cylindrical targets with diameter $12 \mathrm{~mm}$ and height $110 \mathrm{~mm}$ were used. The targets were placed at two different orientations, vertical and $45^{\circ}$ offset from the z-axis. Radiation patterns measurements were performed with the Microwave Scanner from $-120^{\circ}$ to $+120^{\circ}$ in azimuth plane with $20^{\circ}$ increments. The absolute values of cross-polar power levels received at different receiver locations for two different orientations were shown in Fig. 4. The measured
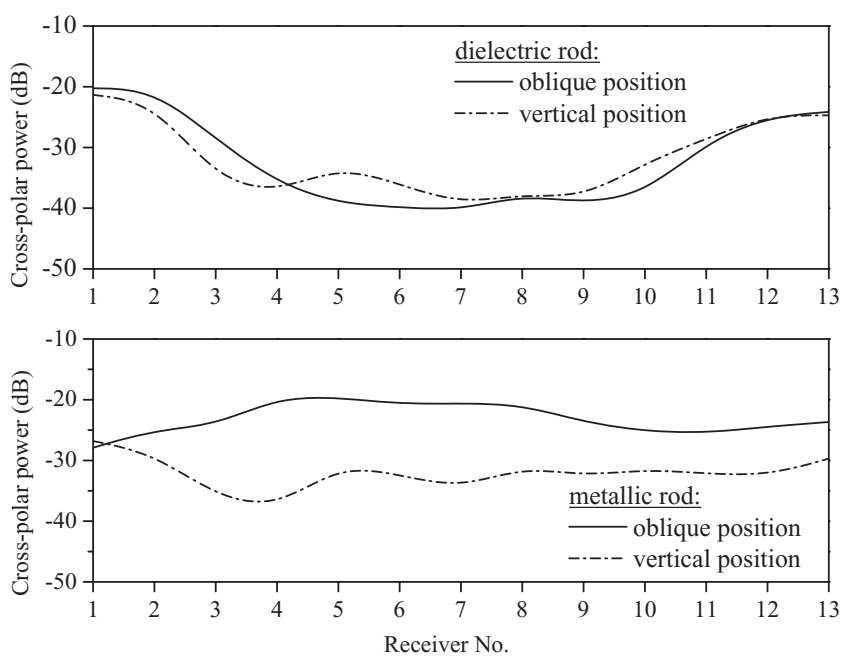

FIG. 4. (top) The cross-polar power level for the dielectric target, (botttom) the cross-polar power level for the metallic target. 
cross-polar components of the dielectric rod and the metallic rod at different locations were shown in top and bottom graph, respectively. The cross-polar components vary significantly for the metallic target (or high Q target) compared to the dielectric target. This variation in cross-polar components will be very useful while performing postprocessing analysis such as angular correlation techniques.

\section{CONCLUSIONS}

This article describes a prototype microwave tomographic scanner for breast cancer detection. The system uses two dual polarized antennas attached to a rotatable platform to measure the angle-dependent scattered radiation fields. A number of LABVIEW virtual instrument modules were developed to control the stepper motors, the VNA, and to archive the measurements in an MDSPlus database. To validate the performance of the scanner, we compared measured data sets with our 3D-FDTD solver, and also the data sets computed by the commercial software packages Ansoft HFSS and CST. Measured behavior is in good agreement with the HFSS simulations. We also confirmed significant differences in the scattered cross-polar power levels for different orientations of high Q target, indicating that vector field measurements will be important for the interpretation of radiation scattered from strongly scattering inhomogeneous targets.

\section{ACKNOWLEDGMENTS}

This work was supported by Australian Research Council ARC Grant No. DP-0667064 and also supported by
VINNOVA within the VINN Excellence Center Chase, and by SSF within the Strategic Research Center, Charmant. The computations were performed on C3SE computing resources at Chalmers University of Technology, Göteborg, Sweden.

${ }^{1}$ A. Fhager and M. Persson, Radio Sci. 40, RS3017 (2005).

${ }^{2}$ J. C. Bolomey, Proc. SPIE 2275, 2 (1994).

${ }^{3}$ Y. J. Kim, L. Jofre, F. D. Flaviis, and M. Q. Feng, IEEE Trans. Microwave Theory Tech. 51(11), 3022 (2003).

${ }^{4}$ T. Takenaka, H. Zhou, and T. Tanaka, J. Opt. Soc. Am. A 20, 1867 (2003).

${ }^{5}$ A. Dubois, K. Belkebirand, and M. Saillard, Inverse Probl. 20, S63 (2004).

${ }^{6}$ Z. Q. Zhang and Q. H. Liu, IEEE Trans. Biomed. Eng. 51, 544 (2004).

${ }^{7}$ S. Y. Semenov, A. E. Bulyshev, A. Abubakar, V. G. Posukh, Y. E. Sizov, A. E. Souvorov, P. M. van den Berg, and T. C. Williams, IEEE Trans. Microwave Theory Tech. 53, 2284 (2005).

${ }^{8}$ R. Nilavalan, A. Gbedemah, I. J. Craddock, X. Li, and S. C. Hagness, Electron. Lett. 39(25), 1787 (2003).

${ }^{9}$ C.-T. Liauh, R. G. Hills, and R. B. Roemer, J. Biomech. Eng. 115, 63 (1993).

${ }^{10}$ M. Gustafsson and S. He, Radio Sci. 35(2), 525 (2000).

${ }^{11}$ T. Tanaka, T. Takenaka, and S. He, Microwave Opt. Technol. Lett. 20(1), 72 (1999).

${ }^{12}$ A. Abubakar and P. M. van den Berg, J. Comput. Phys. 195, 236 (2004).

${ }^{13}$ Q. Fang, P. M. Meaney, S. D. Geimer, A. V. Streltsov, and K. D. Paulsen, IEEE Trans. Med. Imaging 23, 475 (2004).

${ }^{14}$ P. M. Meaney, N. K. Yagnamurthy, and K. D. Paulsen, Phys. Med. Biol. 47, 1101 (2002).

${ }^{15}$ P. M. Meaney, K. D. Paulsen, S. D. Geimer, S. A. Haider, and M. W. Fanning, IEEE Trans. Biomed. Eng. 49(7), 708 (2002).

${ }^{16}$ T. Takenaka, H. Jia, and T. Tanaka, IEICE Trans. Electron. E84-C(12), 1910 (2001).

${ }^{17}$ S. C. Hagness, A. Taflove, and J. E. Bridges, IEEE Trans. Antennas Propag. 47(5), 783 (1999).

${ }^{18}$ S. K. Padhi, A. Fhager, M. Persson, and J. Howard, IEEE Antennas Wirel. Propag. Lett. 7, 689 (2008).

${ }^{19}$ See http://www.mdsplus.org/.

${ }^{20}$ See http://www.cst.com/.

${ }^{21}$ See http://www.ansoft.com/. 